\title{
Comparison of Bruce Treadmill Exercise Test Protocols: Is Ramped Bruce Equal or Superior to Standard Bruce in Producing Clinically Valid Studies for Patients Presenting for Evaluation of Cardiac Ischemia or Arrhythmia with Body Mass Index Equal to or Greater Than 30?
}

\author{
Angela Macci Bires ${ }^{1}$, Dori Lawson ${ }^{2}$, Thomas E. Wasser ${ }^{3}$, and Donna Raber-Baer ${ }^{4}$ \\ ${ }^{1}$ Robert Morris University, Moon Township, Pennsylvania; ${ }^{2}$ Invasive and Non-Invasive Cardiology, Regional Heart Center, The \\ Reading Hospital and Medical Center, West Reading, Pennsylvania; ${ }^{3}$ Consult-Stat: Complete Statistical Services, Macungie, \\ Pennsylvania; and ${ }^{4}$ Regional Heart Center, The Reading Hospital and Medical Center, West Reading, Pennsylvania
}

\begin{abstract}
Clinically valid cardiac evaluation via treadmill stress testing requires patients to achieve specific target heart rates and to successfully complete the cardiac examination. Methods: A comparison of the standard Bruce protocol and the ramped Bruce protocol was performed using data collected over a 1-y period from a targeted patient population with a body mass index (BMI) equal to or greater than 30 to determine which treadmill protocol provided more successful examination results. Results: The functional capacity, metabolic equivalent units achieved, pressure rate product, and total time on the treadmill as measured for the obese patients were clinically valid and comparable to normal-weight and overweight patients $(P<0.001)$. Data gathered from each protocol demonstrated that the usage of the ramped Bruce protocol achieved more consistent results in comparison across all BMI groups in achieving $80 \%-85 \%$ of their age-predicted maximum heart rate. Conclusion: This study did not adequately establish that the ramped Bruce protocol was superior to the standard Bruce protocol for the examination of patients with a BMI equal to or greater than 30 .
\end{abstract}

Key Words: cardiac exercise stress testing protocols; cardiac assessment; Bruce protocol versus ramped Bruce protocol; body mass index (BMI)

J Nucl Med Technol 2013; 41:274-278

DOI: 10.2967/jnmt.113.124727

$\mathbf{T}$ he medical community is well aware of the relationship between obesity, increased heart disease, and decreased life expectancy. The Clinical Trials Services Unit at the University of Oxford, England, conducted a collaborative anal-

\footnotetext{
Received Apr. 12, 2013; revision accepted Oct. 11, 2013.

For correspondence or reprints contact: Angela Macci Bires, Robert Morris University, 6001 University Blvd., Moon Township, PA 16108.

E-mail: bires@rmu.edu

Published online Nov. 12, 2013.

COPYRIGHT (C 2013 by the Society of Nuclear Medicine and Molecular Imaging, Inc.
}

ysis of 57 retrospective studies involving almost 900,000 adults from 1975 through 1985 . This analysis found a positive association between higher mortality and individuals with a body mass index (BMI) equal to 30 or greater. Thus, they concluded that the incidence of mortality directly related to cardiovascular disease was approximately $40 \%$. Mortality increased proportionally with an increase in the BMI. With a BMI of 45 , life expectancy was reduced approximately $8-10$ y because of cardiovascular morbidity in the 2009 Prospective Studies Collection (1).

Cardiovascular evaluations of patients to assess cardiac disease or arrhythmias require valid test parameters. Problems exist with the inability of some patients to achieve $80 \%-85 \%$ of the age-predicted heart rate needed to demonstrate chronotropic competence for diagnostically valid test results (2).

The purpose of this research study was to examine current exercise testing protocols and to determine which exercise protocol is better suited for patients with a BMI equal to 30 or greater. Specifically, this research study investigated the ramped Bruce protocol in comparison to the widely used standard Bruce protocol outlined in Supplemental Appendix 1 (supplemental materials are available at http://tech. snmjournals.org). The ramped Bruce protocol focuses primarily on patients with a BMI equal to or greater than 30 .

The standard Bruce protocol is performed on a treadmill and includes sudden changes in speed and elevation, causing higher oxygen consumption. Because the design of the standard Bruce demands rigorous exertion, patients may stop exercising prematurely, before reaching the required $80 \%-$ $85 \%$ of their age-predicted maximum heart rate (APMHR). Although both the standard and the ramped Bruce protocols are designed for patients to reach the desired maximum heart rate, the ramped Bruce includes more modest changes in speed and elevation, resulting in better patient tolerance (3-7).

In 2001, the American College of Cardiology/American Heart Association (AHA) defined standards for exercise 
testing with treadmills, supporting the use of the ramped Bruce protocol for use with special populations. These standards suggested that the ramped Bruce protocol was both "physiologically and psychologically" preferable for accurate data collection because the 8- to 10-min ramped Bruce protocol would lessen the risk of musculoskeletal injuries and alleviate the anxiety produced when a patient anticipated the next stage increase (2001).

Many studies have compared the standard and ramped Bruce protocols with various patient populations ranging from known healthy individuals to older populations with varying comorbidities $(2-10)$. This literature review was instrumental in establishing the basis of the problem. However, none of these studies gives definitive data confirming the standards sanctioned by the AHA in 2001 regarding proper selection of treadmill protocols for obese patients. In addition, no studies found in the literature specifically focused on comparing protocols to provide justification for the AHA recommendations.

Three studies compared both the standard Bruce and the ramped Bruce protocols and determined that there was no difference in the hemodynamic data obtained from testing with either protocol $(3,4,7)$. Two of the 3 studies also reported that patients had greater tolerance and longer exercise times when testing was performed using the ramped Bruce protocol $(3,4)$.

Dr. Jonathan Myers, using results from the Veterans Specific Activity Questionnaire (VSAQ) in conjunction with a patient's age, created a nomogram to predict the exercise capacity in metabolic equivalents (METs) of a patient undergoing stress testing for cardiovascular disease (7). The VSAQ was a tool developed by Dr. Myers and his group that describes the activities of daily life a patient can perform without symptoms such as shortness of breath, chest pain, or fatigue. One MET is the use of approximately $3.5 \mathrm{~mL}$ of oxygen, per kilogram of body weight, per minute from baseline at rest. When estimating patient capabilities using METs, the AHA in a statement released in 2001, indicated that the ramped Bruce was preferred for accuracy in place of the aggressive standard Bruce protocol.

In conjunction with a research group in Switzerland, Dr. Myers used the VSAQ nomogram to select the proper treadmill protocol for each patient in a group of 43 healthy women. In this study, $100 \%$ of the patients on a ramped Bruce protocol achieve the $80 \%-85 \%$ of the APMHR needed to demonstrate competence for diagnostically valid treadmill time in $8-10 \mathrm{~min}$, whereas only $14 \%$ reached that level on the standard Bruce $(P<0.0001)(8)$.

The ramped Bruce protocol is a consistent, clinically sound procedural parameter needed to generate valid diagnostic testing data during exercise studies. Prognostic variables for individuals with established coronary disease and those who are asymptomatic will affect exercise testing outcomes (9). A vigorous exercise can impair blood flow, and myocardial ischemia can occur (6). Submaximal exercise levels significantly affect myocardial perfusion studies
(10). Across stress laboratories, however, neither consistent exercise protocols nor standard alternative protocols for different populations were found.

\section{MATERIALS AND METHODS}

A retrospective chart review was conducted for a 1-y period within a single testing site. Those charts reviewed included patients aged $18 \mathrm{y}$ and older who underwent a treadmill stress test with or without nuclear testing. Treadmill exercise stress tests performed on patients for the purpose of evaluating cardiac ischemia or arrhythmias using the standard or ramped Bruce protocols were included. All patient chart data were included in our study regardless of the comorbid disease or final diagnostic interpretation. Demographic parameters of the patients included age, sex, weight, height, purpose of examination, BMI, any cardiac medications by class, comorbid conditions, and cardiac history. Exercise data included duration on treadmill, age-predicted heart rate achieved, functional aerobic impairment, and capability measurements in terms of METs. Starting heart rate, peak heart rate, heart rate at $5 \mathrm{~min}$ in recovery, and blood pressure measurements were taken during established protocol exercise stage intervals to calculate pressure rate product (PRP). Final test completion documentation, which included the percentage of the predicted heart rate achieved, was also submitted for analysis and review.

Included in this study were 1,127 charts that were reviewed. Over a 1-y period, all patients who presented for an exercise treadmill study with or without nuclear imaging were included ( $n$ $=1,127$ ). Patients who exercised using the standard Bruce protocol were assigned to the control group $(n=627)$; patients who exercised with the ramped Bruce protocol were the study group ( $n$ $=500$ ). Three patient examination results were eliminated because of incomplete vital exercise data. The patients were grouped by calculated BMI, age, and sex. The groups were broken into subgroups and matched from the control group to the experimental group to reduce bias.

All research personnel handling data reviewed and signed confidentiality agreements, which are filed with the statistical data for a minimum of $5 \mathrm{y}$. All data were collected from a list of patients who were tested in the stress lab at The Reading Hospital and Medical Center Regional Heart Center across a 1-y period. To ensure patient confidentiality, each patient was found within an electronic database and assigned a unique research number. All records are kept in a secure location within the Regional Heart Center for minimum of $5 \mathrm{y}$ from the conclusion of this study. At the end of this time interval, all data will be destroyed.

$\chi^{2}$ tests of association for the dichotomous yes and no data and the other grouped tables, such as group, age, and groupings according to BMI were conducted. For the means comparisons, we examined group $t$ tests between protocol methods. Any $P$ values that were between 0.10 and 0.051 were considered trend significant, and $P$ values that were less than $0.05(P<0.05)$ were considered statistically significant in this study. Because of the exploratory nature of this research, there were no statistical corrections applied to the data for multiple comparisons.

\section{RESULTS}

Both the standard and the ramped Bruce protocols yielded clinically valid studies as defined by the exercise 
TABLE 1

Means Tests on Protocol Versus Raw Age Variable and BMI

\begin{tabular}{lllllllll}
\hline & \multicolumn{3}{c}{ Standard } & & \multicolumn{3}{c}{ Ramped } & \\
\cline { 2 - 4 } Variable & $n$ & Mean & SD & & $n$ & Mean & SD & $P$ \\
\hline BMI & 627 & 30.03 & 6.16 & & 496 & 32.26 & 14.02 & $<0.001$ \\
Age & 627 & 50.80 & 10.41 & & 500 & 63.78 & 11.75 & $<0.001$ \\
\hline
\end{tabular}

variables. However, as BMI increased, the ramped Bruce group had less variation in their functional capacity, METs achieved, PRP, and total time on the treadmill than the standard Bruce group $(P<0.001)$. The ramped Bruce produced more consistent results, and obese patients with BMI of 30 or greater were able to achieve clinically valid studies equally comparable to normal-weight and overweight patients $(P<0.001)$. Three common reasons played a statistically significant role for both normal-weight and overweight patients to end their exercise study in both the standard and the ramped Bruce protocols: dyspnea, fatigue, and leg pain $(P<0.056)$.

The average patient tested on a standard Bruce protocol was $51 \mathrm{y}$ old, was male, and had a BMI of 30.03 (Table 1). In addition, they had less than a $20 \%$ chance of taking a cardiac-related medication (Table 2). The average patient had a heart rate at rest of 70 beats per minute (bpm), which rose to an average of $155 \mathrm{bpm}$ at peak exercise and returned to an average of $92 \mathrm{bpm}$ at the conclusion of $5 \mathrm{~min}$ in recovery. On average, the patient achieved 91\% APMHR, a PRP of $25.71 \mathrm{~K}, 10.67 \mathrm{METs}$, and a functional capacity impairment of $8 \%$ and walked on the treadmill for $9.03 \mathrm{~min}$ $(P<0.001)$ (Table 3).

The average patient tested on the ramped Bruce protocol was male, 64 y old and heavier, with a BMI of 32.26 (Table 4). The patient was more likely to be taking a medication in the $\beta$-blocker class (Table 2). The average resting heart rate was $70 \mathrm{bpm}$, with a peak heart rate of $136 \mathrm{bpm}$ and a 5-min recovery heart rate of $84 \mathrm{bpm}$. On average, the patient achieved $86 \%$ APMHR, a PRP of $22.97 \mathrm{~K}, 8.08$ METs, and a functional capacity impairment of $9 \%$ and walked on the treadmill for $7.39 \min (P<0.001)$ (Table 3).

\section{DISCUSSION}

After data for the entire cohort within each testing protocol were examined, the data were divided into subgroups by age, sex, and BMI ranges. The subgroups focused on the stratification of the BMI groups and the exercise data in each breakdown. On examination of the subgroup exercise data, it became evident that within the standard treadmill protocol an inverse correlation was present between the patient BMI and the amount of time a patient was able to exercise $(P<0.001)$. Specifically, as the patient's BMI increased, the overall time a patient was able to exercise decreased. An increase in functional aerobic impairment was observed between the normal-weight patients with a BMI of 25 or less $(n=112, P=0.019)$ and the obese patients with a BMI of 30 or greater $(n=244, P=0.383)$. These data can be found in detail within Tables 5 and 6 .

Significant differences were observed for both sex and age groups. BMI was also statistically significant between standard and ramped exercise protocols, and BMI was controlled for in the final analysis by making stratifications of BMI $(<25,25-29.99$, and $>30)$. Results of the exercise protocol are reported in Tables 5,6 , and 7 .

Our study results agree with the American College of Cardiology/AHA-defined standards for exercise testing with treadmills. The ramped Bruce protocol outcomes were more consistent, without regard to the patients' BMIs. The exercise data for the normal-weight patients $(n=88)$ (Table 5) and the obese patients $(n=244)$ were almost identical $(P<0.001)$ (Table 6), with the exception of a $2 \%$ decrease in functional aerobic capacity $(P=0.383)$. In addition, the exercise results for the overweight patients (Table 7) $(n=164)$ were also in the same ranges $(P<$ $0.001)$, and their functional capacity was improved by $2 \%$, compared with the normal-weight group $(P=0.141)$.

\section{CONCLUSION}

Both the standard and the ramped Bruce treadmill protocols can result in clinically valid examinations for the evaluation of cardiac ischemia in patients of all body sizes.

TABLE 2

Medication Differences by Protocol

\begin{tabular}{|c|c|c|c|c|c|c|}
\hline \multirow[b]{2}{*}{ Variable } & \multirow[b]{2}{*}{ Value } & \multicolumn{2}{|c|}{ Standard } & \multicolumn{2}{|c|}{ Ramped } & \multirow[b]{2}{*}{$P$} \\
\hline & & Count & Percentage & Count & Percentage & \\
\hline \multirow[t]{2}{*}{$\beta$ Blocker } & No & 397 & 85.2 & 347 & 69.5 & $<0.001$ \\
\hline & Yes & 69 & 14.8 & 152 & 30.5 & \\
\hline \multirow[t]{2}{*}{ ACE/ARB } & No & 375 & 80.5 & 406 & 81.4 & 0.725 \\
\hline & Yes & 91 & 19.5 & 93 & 18.6 & \\
\hline \multirow[t]{2}{*}{ Calcium channel } & No & 543 & 97.2 & 483 & 96.8 & 0.705 \\
\hline & Yes & 13 & 2.8 & 16 & 3.2 & \\
\hline \multirow[t]{2}{*}{ Nitrate } & No & 465 & 99.8 & 496 & 99.4 & 0.350 \\
\hline & Yes & 1 & 0.2 & 3 & 0.6 & \\
\hline \multirow[t]{2}{*}{ None } & No & 174 & 37.3 & 264 & 52.9 & $<0.001$ \\
\hline & Yes & 292 & 62.7 & 235 & 47.1 & \\
\hline
\end{tabular}


TABLE 3

Protocol Test Outcomes All Patients

\begin{tabular}{|c|c|c|c|c|c|c|c|}
\hline \multirow[b]{2}{*}{ Variable } & \multicolumn{3}{|c|}{ Standard } & \multicolumn{3}{|c|}{ Ramped } & \multirow[b]{2}{*}{$P$} \\
\hline & $n$ & Mean & SD & $n$ & Mean & SD & \\
\hline Heart rate, rest & 627 & 70.70 & 12.06 & 500 & 70.72 & 12.31 & 0.979 \\
\hline Heart rate, peak & 627 & 155.15 & 15.45 & 500 & 136.09 & 16.83 & $<0.001$ \\
\hline Heart rate, $5 \mathrm{~min}$ & 627 & 91.99 & 14.19 & 500 & 84.11 & 13.82 & $<0.001$ \\
\hline METs & 627 & 10.67 & 2.59 & 500 & 8.08 & 2.73 & $<0.001$ \\
\hline Maximum predicted heart rate, percentage & 627 & 90.97 & 6.77 & 500 & 86.12 & 10.21 & $<0.001$ \\
\hline PRP & 627 & 25.71 & 4.70 & 500 & 22.97 & 4.92 & $<0.001$ \\
\hline Total treadmill time (s) & 627 & 541.83 & 139.25 & 500 & 443.39 & 137.58 & $<0.001$ \\
\hline Functional capacity impairment & 627 & 7.82 & 10.93 & 500 & 9.05 & 14.51 & 0.103 \\
\hline
\end{tabular}

TABLE 4

$\chi^{2}$ Analysis Protocol Versus Demographics Age Group and Gender

\begin{tabular}{|c|c|c|c|c|c|c|}
\hline \multirow[b]{2}{*}{ Variable } & \multirow[b]{2}{*}{ Value } & \multicolumn{2}{|c|}{ Standard } & \multicolumn{2}{|c|}{ Ramped } & \multirow[b]{2}{*}{$P$} \\
\hline & & Count & Percentage & Count & Percentage & \\
\hline \multirow[t]{2}{*}{ Sex } & Male & 386 & 61.6 & 223 & 44.6 & $<0.001$ \\
\hline & Female & 241 & 38.4 & 277 & 55.4 & \\
\hline \multirow[t]{3}{*}{ Age group } & $18-40$ & 102 & 16.3 & 18 & 3.6 & $<0.001$ \\
\hline & $41-60$ & 409 & 65.2 & 157 & 31.4 & \\
\hline & $61+$ & 116 & 18.5 & 325 & 65.0 & \\
\hline
\end{tabular}

TABLE 5

Protocol Outcomes for Normal-Weight $(\mathrm{BMI}<25)$ Patients

\begin{tabular}{|c|c|c|c|c|c|c|c|}
\hline \multirow[b]{2}{*}{ Variable } & \multicolumn{3}{|c|}{ Standard } & \multicolumn{3}{|c|}{ Ramped } & \multirow[b]{2}{*}{$P$} \\
\hline & $n$ & Mean & SD & $n$ & Mean & SD & \\
\hline Heart rate, rest & 112 & 70.03 & 13.71 & 88 & 70.78 & 13.71 & 0.899 \\
\hline Heart rate, peak & 112 & 158.25 & 17.61 & 88 & 130.61 & 19.24 & $<0.001$ \\
\hline Heart rate, $5 \mathrm{~min}$ & 112 & 89.30 & 17.59 & 88 & 81.73 & 15.46 & $<0.001$ \\
\hline METs & 112 & 11.76 & 2.82 & 88 & 8.04 & 3.00 & 0.002 \\
\hline Maximum predicted heart rate, percentage & 112 & 91.85 & 6.04 & 88 & 85.31 & 11.82 & $<0.001$ \\
\hline PRP & 112 & 24.93 & 4.54 & 88 & 21.19 & 5.09 & $<0.001$ \\
\hline Total time (s) & 112 & 608.67 & 157.46 & 88 & 438.25 & 169.76 & $<0.001$ \\
\hline Functional capacity impairment & 112 & 3.76 & 8.55 & 88 & 8.52 & 17.28 & 0.019 \\
\hline
\end{tabular}

TABLE 6

Protocol Outcomes for Obese $(\mathrm{BMI} \geq 30$ ) Patients

\begin{tabular}{|c|c|c|c|c|c|c|c|}
\hline \multirow[b]{2}{*}{ Variable } & \multicolumn{3}{|c|}{ Standard } & \multicolumn{3}{|c|}{ Ramped } & \multirow[b]{2}{*}{$P$} \\
\hline & $n$ & Mean & SD & $n$ & Mean & SD & \\
\hline Heart rate, rest & 284 & 72.08 & 11.99 & 244 & 70.47 & 12.30 & 0.114 \\
\hline Heart rate, peak & 284 & 153.34 & 13.91 & 244 & 138.65 & 15.04 & $<0.001$ \\
\hline Heart rate, 5 min & 284 & 93.43 & 12.81 & 244 & 85.38 & 13.09 & $<0.001$ \\
\hline METs & 284 & 9.79 & 2.32 & 243 & 7.97 & 2.89 & $<0.001$ \\
\hline Maximum predicted heart rate, percentage & 284 & 89.86 & 6.86 & 244 & 85.49 & 9.68 & $<0.001$ \\
\hline PRP & 284 & 25.99 & 4.45 & 244 & 23.67 & 4.82 & $<0.001$ \\
\hline Total time (s) & 284 & 492.19 & 122.52 & 244 & 435.80 & 123.97 & $<0.001$ \\
\hline Functional capacity impairment & 284 & 11.69 & 12.27 & 244 & 10.69 & 13.93 & 0.383 \\
\hline
\end{tabular}

According to this study, both protocols resulted in patients terminating an examination because of experiencing fatigue, leg pain, and dyspnea. However, there is little difference between the ability to achieve a clinically valid study when exercising a normal-weight patient, an overweight patient, or an obese patient on the ramped Bruce protocol, which was demonstrated to be less physically and mentally imposing for all patients. 
TABLE 7

Protocol Outcomes for Overweight (BMI 25-29.99) Patients

\begin{tabular}{|c|c|c|c|c|c|c|c|}
\hline \multirow[b]{2}{*}{ Variable } & \multicolumn{3}{|c|}{ Standard } & \multicolumn{3}{|c|}{ Ramped } & \multirow[b]{2}{*}{$P$} \\
\hline & $n$ & Mean & SD & $n$ & Mean & SD & \\
\hline Heart rate, rest & 231 & 69.34 & 11.13 & 164 & 71.02 & 13.09 & 0.170 \\
\hline Heart rate, peak & 231 & 155.88 & 15.89 & 164 & 135.56 & 17.17 & $<0.001$ \\
\hline Heart rate, $5 \mathrm{~min}$ & 231 & 91.54 & 13.79 & 164 & 83.41 & 13.80 & $<0.001$ \\
\hline METs & 231 & 11.22 & 2.45 & 164 & 8.30 & 2.29 & $<0.001$ \\
\hline Maximum predicted heart rate, percentage & 231 & 91.92 & 6.82 & 164 & 87.49 & 9.84 & $<0.001$ \\
\hline PRP & 231 & 25.73 & 5.05 & 164 & 22.93 & 4.76 & $<0.001$ \\
\hline Total time (s) & 231 & 570.45 & 127.67 & 164 & 460.99 & 136.28 & $<0.001$ \\
\hline Functional capacity impairment & 231 & 5.01 & 8.36 & 164 & 6.76 & 13.43 & 0.141 \\
\hline
\end{tabular}

When the standard Bruce protocol is used, patients may reach the target heart rate, but they are working harder to do so. Once the BMI of a patient reaches 30 , the patient's functional capacity begins to decrease 3-fold, and the total time of exercise decreases by $20 \%$. Conversely, when the ramped Bruce protocol is used, patients' functional capacities, time on treadmill, and other parameters remain stable regardless of whether their BMI is less than 25 or greater than 30 . We cannot conclude from this study that the appropriate exercise protocol for those with a BMI equal to or greater than 30 is the ramped Bruce.

Additional study is needed with regard to the standard and ramped Bruce protocols. This study serves as a pilot for an additional extensive correlational analysis to begin in the fall 2013. A prospective randomized large-scale study is planned on the basis of the results of this research.

\section{DISCLOSURE}

The costs of publication of this article were defrayed in part by the payment of page charges. Therefore, and solely to indicate this fact, this article is hereby marked "advertisement" in accordance with 18 USC section 1734. Neither the primary investigator, nor any individual who is associated with or responsible for the design, the conduct of, or the reporting of this research study has an economic or financial interest in, or acts as an officer or director for any outside entity whose interests could reasonably appear to be affected by this research project. No potential conflict of interest relevant to this article was reported.

\section{ACKNOWLEDGMENTS}

We thank The Reading Hospital and Medical Center, Robert Morris University DNP faculty, and Barbara Muse Robert Morris University.

\section{REFERENCES}

1. Prospective Studies Collaboration, Whitlock G, Lewington S, et al. Body-mass index and cause-specific mortality in 900000 adults: collaborative analysis of 57 prospective studies. Lancet. 2009;373:1083-1096.

2. Gibbons RJ, Balady GJ, Bricker JT, et al. ACC/AHA 2002 guideline update for exercise testing: summary article: a report of the American College of Cardiology/American Heart Association Task Force on Practice Guidelines (Committee to Update the 1997 Exercise Testing Guidelines). Circulation. 2002;106:18831892 .

3. Will PM, Walter J. Exercise testing: improving performance with a ramped Bruce protocol. Am Heart J. 1999;138:1033-1037.

4. Myers J, Bellin D. Ramp exercise protocols for clinical and cardiopulmonary exercise testing. Sports Med. 2000;30:23-29.

5. Vacanti LJ, Sespedes L, de Oliveria Sarpi, et al. Exercise stress testing is useful, safe and efficient even in patients aged 75 years or older. Arq Bras Cardiol. 2004;82:151-154.

6. Fletcher GF, Balady G, Amsterdam E, et al. Exercise standards for testing and training. Circulation. 2001;104:1694-1740.

7. Myers J, Do D, Herbert W, Ribisl P, Froelicher V. A nomogram to predict exercise capacity from a specific activity questionnaire and clinical data. Am J Cardiol. 1994;73:591-596.

8. Maeder M, Wolber T, Atefy R, et al. A nomogram to select the optimal treadmill ramp protocol in subjects with high exercise capacity: validation and comparison with the Bruce protocol. J Cardiopulm Rehabil. 2006;26:16-23.

9. Mark DB, Lauer M. Exercise capacity: the prognostic variable that doesn't get enough respect. Circulation. 2003;108:1534-1536.

10. Pinkstaff S, Peberdy, MA, Kontos, M, Finucane, S, Arena, R. Quantifying exertion level during stress testing using percentage of age-predicted maximum heart rate, rate pressure product, and perceived exertion. Mayo Clin Proc. 2010; $85: 1095-1100$. 